\title{
A harmonic sum over nontrivial zeros of the Riemann zeta-function*
}

\author{
Richard P. Brent David J. Platt $^{\ddagger}$ and Timothy S. Trudgian ${ }^{\S}$
}

September 14, 2020

\begin{abstract}
We consider the sum $\sum 1 / \gamma$, where $\gamma$ ranges over the ordinates of nontrivial zeros of the Riemann zeta-function in an interval $(0, T]$, and consider the behaviour of the sum as $T \rightarrow \infty$. We show that, after subtracting a smooth approximation $\frac{1}{4 \pi} \log ^{2}(T / 2 \pi)$, the sum tends to a limit $H \approx-0.0171594$ which can be expressed as an integral. We calculate $H$ to high accuracy, using a method which has error $O\left((\log T) / T^{2}\right)$. Our results improve on earlier results by Hassani and other authors.
\end{abstract}

\section{Introduction}

Let the nontrivial zeros of the Riemann zeta-function $\zeta(s)$ be denoted by $\rho=\sigma+i \gamma$. In order of increasing height, the ordinates of these zeros in the upper half-plane are $\gamma_{1} \approx 14.13<\gamma_{2}<\gamma_{3}<\cdots$. Define

$$
G(T):=\sum_{0<\gamma \leqslant T} 1 / \gamma
$$

where multiple zeros (if they exist) are weighted according to their multiplicity. We consider the behaviour of $G(T)$ as $T \rightarrow \infty$. Answering a question of Hassani [7], we show in Theorem 1 of $\$ 2$ that there exists

$$
H:=\lim _{T \rightarrow \infty}\left(G(T)-\frac{\log ^{2}(T / 2 \pi)}{4 \pi}\right) .
$$

\footnotetext{
*Mathematics Subject Classifications: 11M26, 11Y60

${ }^{\dagger}$ Australian National University, Canberra, Australia <Hlimit@rpbrent.com>

${ }^{\ddagger}$ School of Mathematics, University of Bristol, Bristol, UK <dave.platt@bris.ac.uk>

${ }^{\S}$ School of Science, Univ. of NSW, Canberra, Australia <t.trudgian@adfa.edu.au>
} 
There is an analogy with the harmonic series $\sum 1 / n$, which appears in the usual definition of Euler's constant:

$$
C:=\lim _{N \rightarrow \infty}\left(\sum_{n=1}^{N} \frac{1}{n}-\log N\right)=0.577215 \cdots .
$$

It is well-known that one can compute $C$ accurately using Euler-Maclaurin summation or faster algorithms, see [1, 2, 5] and the references given there. However, it is not so easy to compute $H$ accurately, because of the irregular spacing of the nontrivial zeros of $\zeta(s)$, for which see [9].

In $\$$ we consider numerical approximation of $H$, after giving some preliminary lemmas in $\S 3$. If the definition (1) is used directly with the zeros up to height $T$, then the error is $O((\log T) / T)$. In Theorem 2 we show how to improve this, without much extra computation, to $O\left((\log T) / T^{2}\right)$. In Corollary 1 we give an explicit bound on $H$ with error of order $10^{-18}$.

Finally, in $\$ 5$, we comment briefly on related results in the literature.

\section{Existence of the limit}

Before proving Theorem 1, we define some notation. Let $\mathcal{F}$ denote the set of positive ordinates of zeros of $\zeta(s)$. Following Titchmarsh [11, $\S 9.2-$ $\S 9.3]$, if $0<T \notin \mathcal{F}$, then we let $N(T)$ denote the number of zeros $\beta+i \gamma$ of $\zeta(s)$ with $0<\gamma \leqslant T$, and $S(T)$ denote the value of $\pi^{-1} \arg \zeta\left(\frac{1}{2}+i T\right)$ obtained by continuous variation along the straight lines joining $2,2+i T$, and $\frac{1}{2}+i T$, starting with the value 0 . If $T \in \mathcal{F}$, we could take $S(T)=$ $\lim _{\delta \rightarrow 0}[S(T-\delta)+S(T+\delta)] / 2$, and similarly for $N(T)$, but we avoid this exceptional case. Note that $N(T)$ and $S(T)$ are piecewise continuous, with jumps at $T \in \mathcal{F}$.

By [11, Thm. 9.3], we have $N(T)=L(T)+Q(T)$, where

$$
L(T)=\frac{T}{2 \pi}\left(\log \left(\frac{T}{2 \pi}\right)-1\right)+\frac{7}{8}, \text { and } Q(T)=S(T)+O(1 / T) .
$$

An explicit bound from Trudgian [13, Cor. 1] is

$$
Q(T)=S(T)+\frac{0.2 \vartheta}{T}
$$

where (here and elsewhere) $\vartheta \in \mathbb{R}$ satisfies $|\vartheta| \leqslant 1$.

Let $S_{1}(T):=\int_{0}^{T} S(t) d t$. By [11, Thm. 9.4 and Thm. 9.9(A)], we have $S(T)=O(\log T)$ and $S_{1}(T)=O(\log T)$, and it follows from (2) that $Q(T)=O(\log T)$ also. 
Explicit bounds on $S_{1}(T)$ are known. For certain constants $c, A_{0} \geqslant 0$, $A_{1} \geqslant 0$, and $T_{0}>0$, there is a bound

$$
\left|S_{1}(T)-c\right| \leqslant A_{0}+A_{1} \log T \text { for all } T \geqslant T_{0} .
$$

From [12, Thm. 2.2], we could take $c=S_{1}(168 \pi), A_{0}=2.067, A_{1}=0.059$, and $T_{0}=168 \pi$. However, a small computation shows that (3) also holds for $T \in[2 \pi, 168 \pi]$. Hence, we take $T_{0}=2 \pi$ in $(3)$.

Our first result is the following.

Theorem 1. The limit $H$ in (1) exists. Also,

$$
H=\int_{2 \pi}^{\infty} \frac{Q(t)}{t^{2}} d t-\frac{1}{16 \pi}
$$

where $Q(T)=N(T)-L(T)$ is as above.

Proof. Suppose that $2 \pi \leqslant T \notin \mathcal{F}$. Using Stieltjes integrals, and noting that $\gamma_{1}>2 \pi$ and $Q(2 \pi)=\frac{1}{8}$, we have

$$
\begin{aligned}
G(T) & =\sum_{0<\gamma \leqslant T} \frac{1}{\gamma}=\int_{2 \pi}^{T} \frac{d N(t)}{t}=\int_{2 \pi}^{T} \frac{d L(t)}{t}+\int_{2 \pi}^{T} \frac{d Q(t)}{t} \\
& =\frac{1}{2 \pi} \int_{2 \pi}^{T} \frac{\log (t / 2 \pi)}{t} d t+\left[\frac{Q(t)}{t}+\int \frac{Q(t)}{t^{2}} d t\right]_{2 \pi}^{T} \\
& =\frac{\log ^{2}(T / 2 \pi)}{4 \pi}+\frac{Q(T)}{T}-\frac{1}{16 \pi}+\int_{2 \pi}^{T} \frac{Q(t)}{t^{2}} d t .
\end{aligned}
$$

Thus

$$
G(T)-\frac{\log ^{2}(T / 2 \pi)}{4 \pi}=\int_{2 \pi}^{T} \frac{Q(t)}{t^{2}} d t-\frac{1}{16 \pi}+O((\log T) / T) .
$$

Letting $T \rightarrow \infty$, the last integral converges, so the limit of the left-hand-side exists, and we obtain

$$
H=\lim _{T \rightarrow \infty}\left(G(T)-\frac{\log ^{2}(T / 2 \pi)}{4 \pi}\right)=\int_{2 \pi}^{\infty} \frac{Q(t)}{t^{2}} d t-\frac{1}{16 \pi} .
$$

This completes the proof. 


\section{Two lemmas}

We now give two lemmas that are used in the proof of Theorem 2 .

Lemma 1. If $2 \pi \leqslant T \notin \mathcal{F}$, then

$$
\int_{2 \pi}^{T} \frac{Q(t)}{t^{2}} d t=G(T)-\frac{Q(T)}{T}+\frac{1}{16 \pi}-\frac{\log ^{2}(T / 2 \pi)}{4 \pi} .
$$

Proof. This is just a rearrangement of (4) in the proof of Theorem 1 .

Lemma 2. If $T \geqslant 2 \pi$ and

$$
E_{2}(T):=\int_{T}^{\infty} \frac{Q(t)}{t^{2}} d t
$$

then

$$
\left|E_{2}(T)\right| \leqslant \frac{4.27+0.12 \log T}{T^{2}} .
$$

Proof. To bound $E_{2}(T)$ we note that, from (2),

$$
\int_{T}^{\infty} \frac{Q(t)}{t^{2}} d t=\int_{T}^{\infty} \frac{S(t)}{t^{2}} d t+\frac{0.1 \vartheta}{T^{2}}
$$

Also, using integration by parts,

$$
\int_{T}^{\infty} \frac{S(t)}{t^{2}} d t=-\frac{S_{1}(T)-c}{T^{2}}+2 \int_{T}^{\infty} \frac{S_{1}(t)-c}{t^{3}} d t
$$

Using (3), we have

$$
\begin{aligned}
\left|\int_{T}^{\infty} \frac{S(t)}{t^{2}} d t\right| & \leqslant \frac{\left|S_{1}(T)-c\right|}{T^{2}}+2 \int_{T}^{\infty} \frac{\left|S_{1}(t)-c\right|}{t^{3}} d t \\
& \leqslant \frac{A_{0}+A_{1} \log T}{T^{2}}+2 \int_{T}^{\infty} \frac{A_{0}+A_{1} \log t}{t^{3}} d t \\
& =\frac{2 A_{0}+0.5 A_{1}+2 A_{1} \log T}{T^{2}} .
\end{aligned}
$$

Using (6), this gives

$$
\left|E_{2}(T)\right| \leqslant \frac{2 A_{0}+0.5 A_{1}+0.1+2 A_{1} \log T}{T^{2}} .
$$

Inserting the values $A_{0}=2.067$ and $A_{1}=0.059$ gives the result. 
We note that the bound (8) might be improved by using a result of Fujii [6. Thm. 2] to bound the integral of $S_{1}(t) / t^{3}$ in (7), although we are not aware of any explicit version of Fujii's estimate. The bound would then be dominated by the term $-S_{1}(T) / T^{2}$ in (7). This term is $o\left((\log T) / T^{2}\right)$ iff the Lindelöf Hypothesis (LH) is true, see [11, Thm 13.6(B) and Note 13.8]. Thus, obtaining an order-of-magnitude improvement in the bound on $E_{2}(T)$ is equivalent to proving $\mathrm{LH}$.

\section{Numerical approximation of $H$}

We consider two methods to approximate $H$ numerically. The first method truncates the sum and integral in the definition (1) at height $T \geqslant 2 \pi e$, giving an approximation with error $E(T)=O((\log T) / T)$. An explicit bound

$$
H=G(T)-\frac{\log ^{2}(T / 2 \pi)}{4 \pi}+A \vartheta\left(\frac{2 \log T+1}{T}\right)
$$

follows from Lehman [8, Lem. 1]. Lehman gave $A=2$, but from [3, Cor. 1] we may take $A=0.28$. Thus, we can obtain about 5 decimal places by summing over the first $10^{6}$ zeros of $\zeta(s)$, i.e. to height $T=600270$. In this manner we find $H \approx-0.01716$. It is difficult to obtain many more correct digits because of the slow convergence. However, the result is sufficient to show that $H$ is negative, which is significant in the proof of [3, Lem. 8].

Convergence can be accelerated using Theorem 2, which improves the error bound $E(T)=O((\log T) / T)$ of 9 to $E_{2}(T)=O\left((\log T) / T^{2}\right)$. Note that the error term $E_{2}(T)$ is a continuous function of $T$. This is unlike $E(T)$, which has jumps for $T \in \mathcal{F}$.

Theorem 2. For all $T \geqslant 2 \pi$,

$$
H=\sum_{0<\gamma \leqslant T}\left(\frac{1}{\gamma}-\frac{1}{T}\right)-\frac{\log ^{2}(T / 2 \pi e)+1}{4 \pi}+\frac{7}{8 T}+E_{2}(T),
$$

where $E_{2}(T)$ is as in (5), and $\left|E_{2}(T)\right| \leqslant(4.27+0.12 \log T) / T^{2}$.

Proof. First assume that $T \notin \mathcal{F}$. From Theorem 1 and Lemma 1 ,

$$
H=G(T)-\frac{Q(T)}{T}-\frac{\log ^{2}(T / 2 \pi)}{4 \pi}+E_{2}(T),
$$

but $Q(T)=N(T)-L(T)$, so

$$
H=\sum_{0<\gamma \leqslant T}\left(\frac{1}{\gamma}-\frac{1}{T}\right)+\frac{\log (T / 2 \pi)-1}{2 \pi}+\frac{7}{8 T}-\frac{\log ^{2}(T / 2 \pi)}{4 \pi}+E_{2}(T) .
$$


Simplification gives (10), and a continuity argument shows that (10) holds if $T \in \mathcal{F}$. Finally, the bound on $E_{2}(T)$ follows from Lemma 2 ,

Corollary 1. Let $H$ be defined by (1). We have

$$
H=-0.0171594043070981495+\vartheta\left(10^{-18}\right) .
$$

Proof. This follows from Theorem 2 by an interval-arithmetic computation using the first $n=10^{10}$ zeros, with $T=\gamma_{n} \approx 3293531632.4$.

To illustrate Theorem 2, we give some numerical results in Table 1. The first column $(n)$ gives the number of zeros used, and the second column is the estimate of $H$ obtained from (10), using $T=\gamma_{n}$. The first incorrect digit of each entry is underlined.

\begin{tabular}{|c|c|}
\hline$n$ & $H$ estimate \\
\hline 10 & $-0.017 \underline{3} 72393877$ \\
100 & $-0.017159 \underline{7} 65533$ \\
1000 & $-0.017159 \underline{6} 03500$ \\
10000 & $-0.017159404 \underline{8} 75$ \\
100000 & $-0.017159404 \underline{2} 44$ \\
1000000 & -0.017159404307 \\
\hline
\end{tabular}

Table 1: Numerical estimation of $H$ using Theorem 2 ,

\section{Related results in the literature}

Büthe [4, Lem. 3] gives the inequality

$$
G(T) \leqslant \frac{\log ^{2}(T / 2 \pi)}{4 \pi} \text { for } T \geqslant 5000 .
$$

In [3, Lem. 8], we give a different proof of (11), and show that it holds for $T \geqslant 4 \pi e$.

Hassani [7] shows (in our notation) that

$$
G(T)=\frac{\log ^{2}(T / 2 \pi)}{4 \pi}+O(1)
$$

and gives numerical bounds for the "O(1)" term. A similar bound is given in [10, Lem. 2.10]. Hassani does not prove existence of the limit (1), but asks (see [7, p. 114]) whether it exists. We have answered this in our Theorem 1. 
In fact, Hassani works with

$$
\Delta_{N}:=\sum_{n=1}^{N} \frac{1}{\gamma_{n}}-\left(\frac{1}{4 \pi} \log ^{2} \gamma_{N}-\frac{\log (2 \pi)}{2 \pi} \log \gamma_{N}\right),
$$

so in our notation

$$
\Delta_{N}=G\left(\gamma_{N}\right)-\frac{\log ^{2}\left(\gamma_{N} / 2 \pi\right)}{4 \pi}+\frac{\log ^{2}(2 \pi)}{4 \pi} .
$$

Thus, the (hypothetical) limit to which Hassani refers is, in our notation,

$$
H+\frac{\log ^{2}(2 \pi)}{4 \pi}=0.2516367513127059665+\vartheta\left(10^{-18}\right) .
$$

This is consistent with the value 0.25163 that Hassani gives based on his calculations using $2 \cdot 10^{6}$ nontrivial zeros. Hassani also uses an averaging technique to obtain values in the range [0.2516372, 0.2516375], but apparently decreasing, without an obvious limit. The acceleration technique of Theorem 2 is more effective, and has the virtue of giving a rigorous error bound.

\section{Acknowledgements}

TST is supported by ARC Grants DP160100932 and FT160100094; DJP is supported by ARC Grant DP160100932 and EPSRC Grant EP/K034383/1.

\section{References}

[1] R. P. Brent and F. Johansson, A bound for the error term in the BrentMcMillan algorithm, Math. Comp. 84(295):2351-2359, 2015.

[2] R. P. Brent and E. M. McMillan, Some new algorithms for highprecision computation of Euler's constant, Math. Comp. 34(149):305312, 1980.

[3] R. P. Brent, D. J. Platt, and T. S. Trudgian, The mean square of the error term in the prime number theorem, submitted, 2020. Preprint available at arXiv:2008.06140v1.

[4] J. Büthe, Estimating $\pi(x)$ and related functions under partial RH assumptions, Math. Comp. 85(301):2483-2498, 2016. 
[5] J.-P. Demailly, Precise error estimate of the Brent-McMillan algorithm for the computation of Euler's constant, Mosc. J. Comb. Number Theory $7(4): 271-306,2017$.

[6] A. Fujii, On the zeros of the Riemann zeta function, Comment. Math. Univ. St. Paul. 51(1):1-17, 2002.

[7] M. Hassani, Explicit approximation of the sums over the imaginary part of the non-trivial zeros of the Riemann zeta function, Appl. Math. E-Notes 16:109-116, 2016.

[8] R. S. Lehman, On the difference $\pi(x)-\operatorname{li}(x)$, Acta Arith. 11:397-410, 1966.

[9] A. M. Odlyzko, On the distribution of spacings between zeros of the zeta function, Math. Comp. 48(177):273-308, 1987.

[10] Y. Saouter, T. Trudgian, and P. Demichel, A still sharper region where $\pi(x)-\operatorname{li}(x)$ is positive, Math. Comp. 84(295):2433-2446, 2015.

[11] E. C. Titchmarsh, The Theory of the Riemann Zeta-function, 2nd ed. (edited and with a preface by D. R. Heath-Brown), Oxford, 1986.

[12] T. S. Trudgian, Improvements to Turing's method, Math. Comp. 80(276):2259-2279, 2011.

[13] T. S. Trudgian, An improved upper bound for the argument of the Riemann zeta-function on the critical line II, J. Number Theory 134:280$292,2014$. 